\title{
Impact of Hepatic Stellate Cells in Scaffold-Free 3D-Bioprinting of the Liver Model
}

\author{
Cutter J. Koehler ${ }^{1}$, Wenjun Zhang ${ }^{1}$, Julia R. Walsh ${ }^{1}$, Raza A. Naqvi ${ }^{1}$, Erika \\ Gramelspacher ${ }^{1}$, Lester J. Smith ${ }^{2,3}$, Ping Li ${ }^{1}$, Burcin Ekser ${ }^{1}$
}

(1) Transplant Surgery, Department of Surgery, Indiana University School of Medicine; (2) 3D-Bioprinting Core, Indiana University School of Medicine; (3)

Department of Radiology and Imaging Sciences, Indiana University School of Medicine

Background and Hypothesis: Hepatic stellate cells (HSC), which compromise $\sim 15 \%$ of liver cells, are vital to hepatocellular function. Scaffold-free 3Dbioprinting (SF3DBP) offers an avenue for the creation of realistic organ models without the use of biomaterials. Therefore, we hypothesized that co-culturing primary hepatocytes with HSC in SF3DBP liver model would uphold hepatocyte function over time, providing us a better 3D-liver model for research.

Experimental Design: We used freshly thawed primary pig hepatocytes and immortalized pig HSC to generate spheroids with hepatocytes alone, HSC alone, or a combination of hepatocytes and HSC (2.5:1 ratio). Spheroids were formed using low adhesion plates, then characterized for distance from well center, diameter, roundness, and smoothness. A column of spheroids was printed using a Regenova 3D-bioprinter. Remaining loose spheroids are incubated over two weeks for albumin secretion, mRNA transcription, and histological analysis.

Results: Co-cultures of hepatocytes and HSC (2.5:1 ratio) formed spheroids within 48 hours, as did HSC only spheroids (Figure 1). Spheroids composed of only hepatocytes failed to form round spheroids. The combination spheroids increased in roundness and decreased in diameter between characterizations over 6 days.

Conclusion and Potential Impact: Spheroids proved too large to print at 48 hours but were successfully recognized and placed by the 3D-bioprinter. SF3DBP of combination spheroids would be viable by day 6 . Optimization of spheroid composition using different cell ratios including HSC, hepatocytes, liver sinusoidal endothelial cells and fibroblasts, as well as optimization of spheroid incubation time will allow for production and printing of more advanced liver models. 
Figure 1:

\section{Pig Liver HSC \\ Pig Liver HC \\ Pig Liver 2.5HC:1HSC}

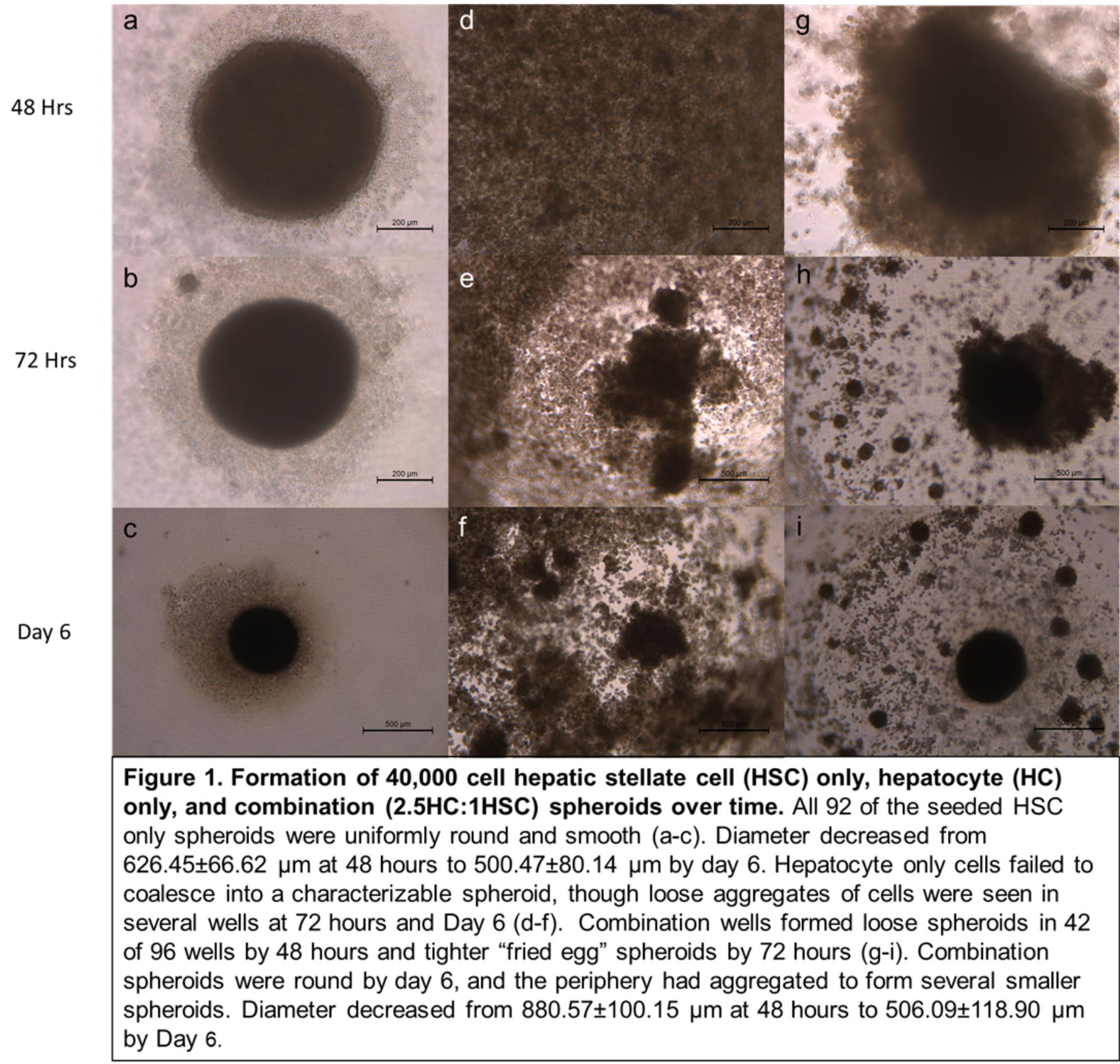

